\title{
Phosphorescence of CdS nanoparticles in polymer matrix as an indication of host-guest interaction
}

\author{
A.O. Kovalchuk ${ }^{\text {a, }}{ }^{*}$, G.Yu. Rudko a, V.I. Fediv ${ }^{\text {b }}$, W.M. Chen ${ }^{\text {c }}$, I.A. Buyanova ${ }^{c}$ \\ ${ }^{a}$ V. Lashkaryov Institute of Semiconductor Physics of National Academy of Sciences of Ukraine, 45, Pr. Nauky, Kyiv, 03028, Ukraine \\ ${ }^{\mathrm{b}}$ Department of Biophysics and Medical Informatics, Bukovinian State Medical University, 42 Kobylyanska St., 58000, Chernivtsi, Ukraine \\ ${ }^{\mathrm{c}}$ Department of Physics, Chemistry and Biology, Linköping University, SE-581 83, Linköping, Sweden
}

\section{H I G H L I G H T S}

- Long-lasting low-temperature photoluminescence decay in nano-CdS/ PVA is observed.

- Characteristic time about $1.7 \mathrm{~s}$ for photoluminescence decay is measured.

- Accumulation of photo-excited carriers in traps within the PVA is shown.

- Transfer of the excitation from PVA to CdS nanoparticles is shown.

\section{A R T I C L E I N F O}

\section{Article history:}

Received 21 February 2016

Received in revised form

5 April 2016

Accepted 11 April 2016

Available online $\mathrm{xxx}$

\section{G R A P H I C A L A B S T R A C T}

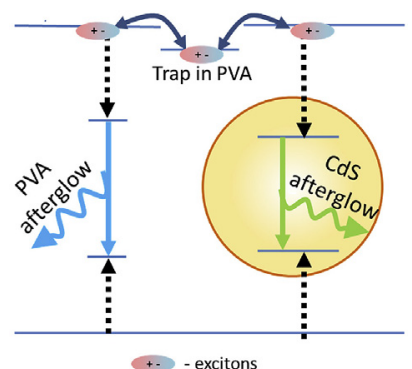

A B S T R A C T

We report on the observation of the long-lasting low-temperature photoluminescence decay in the hybrid system nano-CdS/polyvinyl alcohol with a characteristic time of about $1.7 \mathrm{~s}$. The origin of the phosphorescence is ascribed to the accumulation of photo-excited excitons in the traps within the polymeric matrix with subsequent transfer of the excitation to the embedded CdS nanoparticles.

() 2016 Elsevier B.V. All rights reserved.

\section{Keywords:}

Composite materials

Nanostructures

Photoluminescence spectroscopy

Semiconductors

\section{Introduction}

Light-emitting properties of semiconductor nanoparticles (NPs) attract vigorous interest due to a high efficiency and wide tunability of color that makes them promising for applications in the fields of optoelectronics, photonics, biosensing, etc. (see the reviews [1-3]

\footnotetext{
* Corresponding author. Tel.: +380 976694174.

E-mail address: andriy_kovalchuk@yahoo.com (A.O. Kovalchuk).
}

and references therein). By incorporation NPs into various matrices and, hence, formation of nanocomposites, new functionalities can be obtained. Impacts on the materials properties can be achieved both by combining the benefits of NPs with the advantages of host materials and by enabling host-guest interactions. The latter include an exchange of charged particles between the NPs and matrices, bi-lateral energy transfer processes, formation of new bonding at the interface NP/host, etc [4-6]. All these factors can vary the intensity, spectral and time characteristics of light emitted by the NPs. In Refs. [7,8] an alteration of NPs' luminescence spectral 
characteristics and intensity due to the host material influence were reported. However, to the best of our knowledge, there are no reports on the phosphorescence in semiconductor NPs caused by interactions with the enclosing material.

Generally, phosphorescence is not a common occurrence in semiconductor NPs while it is frequently observed in other objects. This phenomenon is typical for the luminescence of molecules, when the emission occurs via the forbidden transitions [9]. Another characteristic example is the afterglow of bulk crystal phosphors, where the long-lasting emission decay is caused by the presence of the traps that accommodate the photoexcited carriers for a prolonged time until they are thermally released and then recombine radiatively [for the review, see 10 ].

To the best of our knowledge, there are only three observations of the phosphorescence of NPs. In Refs. [11,12] the observation of phosphorescence in the time scale of milliseconds was reported in Mn-doped ZnS NPs, where the radiative recombination arises from the transition between the excited triplet state ${ }^{4} \mathrm{~T}_{1}$ of $\mathrm{Mn}^{2+}$ ion and its singlet ground state ${ }^{6} \mathrm{~A}_{1}$. Much longer afterglow (with the decay time of about a second) was observed in ZnS NPs embedded in the glass matrix [13]; however, in the latter paper the origin of the phenomenon was not analyzed.

In the present paper, we report on the observation of longlasting low-temperature phosphorescence of CdS NPs embedded in polyvinyl alcohol (PVA) polymer and analyze the role of the polymer matrix in the processes of excitation and emission of light.

\section{Materials and methods}

Colloidal CdS NPs were synthesized in a water solution of PVA as have been described in detail in Ref. [14]. Molecules of PVA served as capping agents that restricted the growth of the NPs and prevented their coagulation. PVA also served as a film-forming matrix to obtain solid flexible films accommodating the light-emitting NPs.

A starting solution for the growing NPs contained 5 (wt.)\% of PVA. This commercial PVA had a molecular weight close to 50,000 . The salts $\mathrm{CdCl}_{2}$ and $\mathrm{Na}_{2} \mathrm{~S}$ were used as the precursors for NPs growth. Salts concentrations as well as a $\mathrm{pH}$ value of the solution $(\mathrm{pH}=4.8)$ were chosen to prevent the formation of $\mathrm{Cd}(\mathrm{OH})_{2} \cdot \mathrm{pH}$ value was adjusted by adding $0.1 \mathrm{M} \mathrm{HCl}$ solution to the growth medium. The final product of the synthesis procedure is a colloidal solution of the CdS NPs. An average diameter of the NPs is $5.4 \mathrm{~nm}$ (a typical transmission electron microscope image can be seen in Ref. [15], Fig. 1). The NPs were crystalline with the wurtzite type of crystal lattice [16].

To obtain thin solid films of composites and reference samples of the unloaded PVA, equal volumes of colloidal solutions were dried in glass Petri dishes. These dishes were placed into a closed vessel containing absorbent $\mathrm{CaCl}_{2}$ that absorbed moisture from the air inside the vessel. All samples preparation steps were done at ambient temperature and pressure. The structure of the polymeric matrix of solid nanocomposite films as well as of the reference unloaded polymeric samples was partly amorphous and partly crystalline which is quite typical for PVA [16]. It should be noted that the crystallinity of the polymeric matrix in these nanocomposites is somewhat higher due to the increased amount of hydrogen bonds.

Photoluminescence (PL) of the CdS/PVA nanocomposite and PVA polymer was excited using a Coherent Verdi $2 \mathrm{~W}$ laser operating at $\lambda_{\text {exc }}=266 \mathrm{~nm}$. PL signals were registered by a CCD camera (Princeton Instruments ST-133) assembled with an Acton SpectraPro $2500 \mathrm{i}$ monochromator. The experiments were done at the temperature of $5 \mathrm{~K}$.

Time-delayed spectra of the CdS/PVA composite and pure PVA

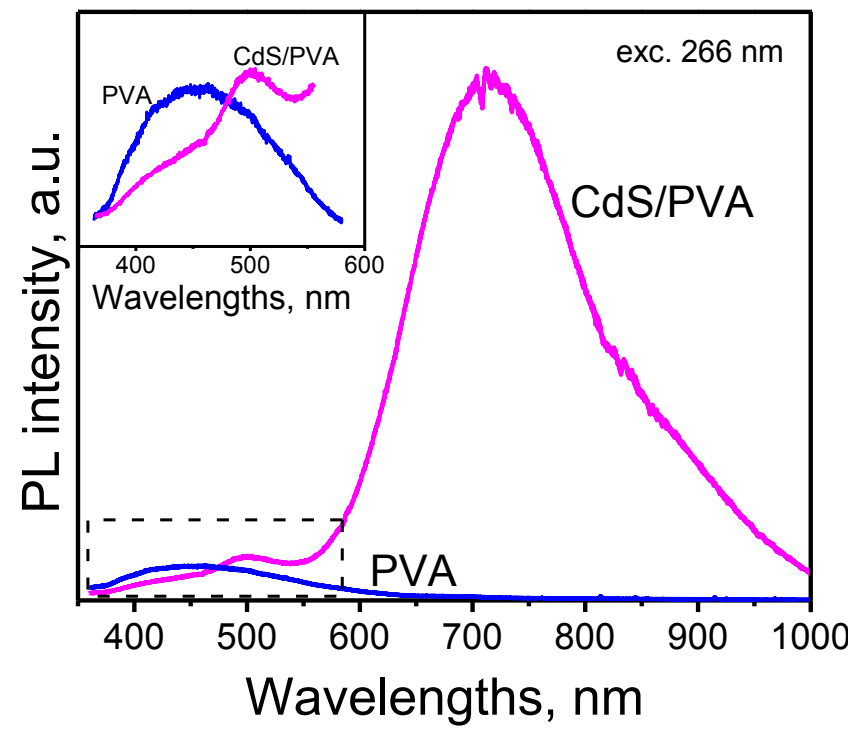

Fig. 1. cw PL spectra of nano-CdS/PVA composite and the unloaded PVA. T $=5 \mathrm{~K}$.

were recorded with the same CCD camera that was used for $\mathrm{cw}$ spectra registration. After switching-off the laser, series of PL spectra were recorded with the $0.5 \mathrm{~s}$ acquisition time for each spectrum and the interval of $1 \mathrm{~s}$ between the beginnings of the measurements.

\section{Results}

Fig. 1 shows PL spectra of the CdS/PVA nanocomposite and pure PVA. The spectral range $350-600 \mathrm{~nm}$ is shown in a larger scale in the inset. Extremely wide and intense band with the maximum at $\sim 720 \mathrm{~nm}$ and the wing in the near infrared spectral range dominates the PL spectrum of the composite. Much narrower and less intense band is seen at $\sim 510 \mathrm{~nm}$. This band has a wing in the short wavelengths range. By comparing with the emission spectrum of the pure PVA (see the inset in Fig. 1), this wing can be attributed to the presence of a residual emission from the polymeric matrix in the spectrum of the nanocomposite. Notably, the emission of the matrix is reduced in the composite. We ascribe this quenching of the matrix PL emission in the composite partially to the reabsorption by the NPs and partially to the excitation transfer from the matrix to the incorporated NPs that has been previously demonstrated by the time-decay measurements in the nanosecond range [15].

The observed type of the PL spectra is quite typical for CdS nanocrystals. Usually, the emission of the CdS NPs in the green and red regions is ascribed to shallow [17,18] and deep [19] traps. The spectrum of polymeric matrix - the asymmetric wide band with the maximum at $460 \mathrm{~nm}$ - is also typical for PVA, and is usually ascribed to carbonyl-related chromophore groups that are inherent to commercial PVA [20].

Fig. 2,a demonstrates the long-lasting afterglow in nano-CdS/ PVA composite. The results for the unloaded PVA are shown in Fig. 2,b for a comparison. Note, that the long-lasting phosphorescence was reliably observed only in the blue-green spectral range (380-550 nm) while the emission in the red and near infrared ranges faded much faster and, thus, could not be unambiguously registered within the limits of the error of the experimental set-up used. Therefore, hereafter only the blue-green region will be discussed.

The curves in Fig. 2,a,b demonstrate PL spectra of the samples 

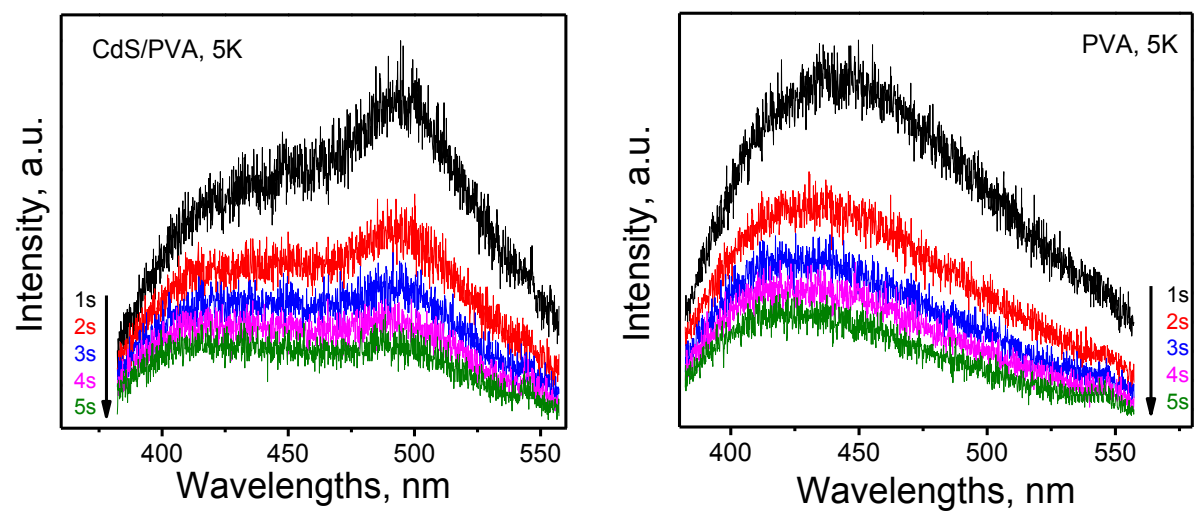

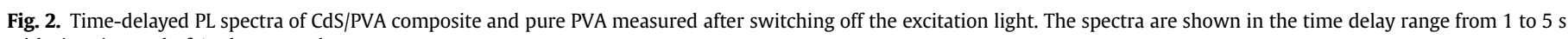
with time interval of $1 \mathrm{~s}$ between the spectra.

measured sequentially after switching off the excitation light starting with $1 \mathrm{~s}$ delay. The value of the delay is labeled on the figures. As is seen for the CdS/PVA nanocomposite, both PL features that are observed under the cw excitation in the blue-green region are also seen in the time-delayed spectra.

Fig. 3 represents the time dependencies of the PL intensities registered at the maxima of the PL features shown in the inset in Fig. 1, namely, the measurements were done at $510 \mathrm{~nm}$ for the CdS/ PVA composite and at $460 \mathrm{~nm}$ for the pure PVA. The intensity is plotted in the logarithmic scale to show the low-intensity range in more detail. The kinetics curves for both structures cannot be fitted by a mono-exponential law. Such processes can be characterized only by an average decay time, i.e. the time that is required for $e$ times decrease in the initial intensity. It is seen that the value of average decay time is about $1.7 \mathrm{~s}$ and is the same for both samples.

\section{Discussion}

To the best of our knowledge, this is the first observation of the average emission decay time as large as $1.7 \mathrm{~s}$ for CdS/PVA nanocomposite. Typically, PL decay times for such systems are in the

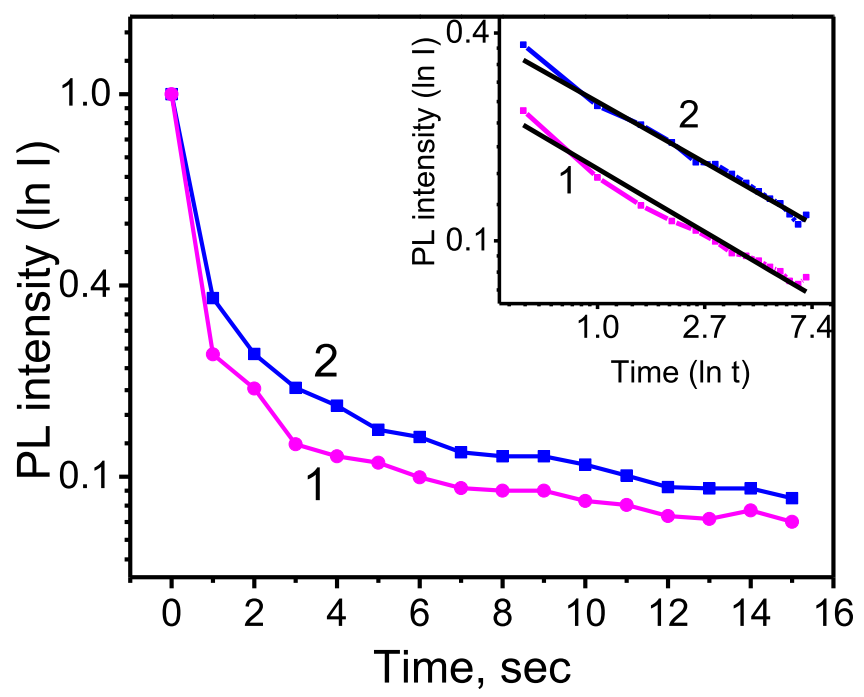

Fig. 3. Time decays of the PL intensity after switching off the excitation light measured at the maximum of the cW PL band: 1 - the nanocomposite CdS/PVA, registration at $510 \mathrm{~nm}, 2$ - the pure PVA, registration at $460 \mathrm{~nm}$. The inset shows the same experimental results in the double logarithmic scale and fitting by the Becquerel formula (the solid straight lines). range from nanoseconds [21] to microseconds [22]. As was stated above, the observed PL decays for both composite and pure PVA are not exponential (Fig. 3). Such long-lasting non-exponential decays are observed for the phosphorescence in crystal phosphors. In this case, the afterglow is caused by accumulation of photo-excited carriers on certain trap states during sample illumination. After the excitation is switched off, the carriers are thermally released from these traps and emit light via recombination at radiative centers. For the description of the corresponding decay kinetics Becquerel has proposed [23] the empirical formula

$I_{/} I_{0}=1 /(1+W t)^{\alpha}$

where $I_{0}$ is the intensity under cw excitation, $t$ is the time, and $\alpha$ and $\mathrm{W}$ are the experimentally determined parameters. The classical interpretation of the hyperbolic decay of luminescence has been proposed by Fok for the description of the phosphorescence of crystal phosphors [for the review, see 10]. The model accounted for the presence of both light-emitting centers and traps for photoexcited carriers in a non-emitting matrix. Under the assumption that only one type of emitting centers and only one type of traps are present, the hyperbolic decay with $\alpha=2$ was obtained.

To check the applicability of hyperbolic law to the description of the afterglow curves of composite and pure PVA, we have replotted the experimental data in the double logarithmic scale (see the inset in Fig. 3) and fitted them using the formula (1) (the fitting results are shown by the solid straight lines). The best fit to the experimental data can be obtained assuming that the hyperbolic indexes $\alpha$ for CdS/PVA composite and pure PVA are the same and equal to 0.3 , whereas the $W$ coefficients are equal to 210 and 90 for CdS/PVA and PVA, respectively. It should be noted that the deduced hyperbolic indexes are different from the value $\alpha=2$ obtained by the simplest theory [10]. They are also less than the value $1<\alpha<2$ that is predicted by a more complicated theory that accounts for two trap levels and two radiative centers [10]. However, such a discrepancy between experimental results and theoretical predictions is quite usual. Afterglow curves that obey hyperbolic laws with indexes $\alpha=0.25$, and $\alpha=0.4$ were observed for $\mathrm{ZnMoO}_{4}$ crystals [24], which points to a more complicated ensemble of trapping and emitting energy levels than the ones discussed by classical theory as well as to the involvement of nonradiative processes. It should be also noted that in none of the cases the hyperbolic law could describe the experimental decay curves in the whole time range.

The above observations suggest that the origin of the decay must be common both for nanocomposite CdS/PVA and unloaded 

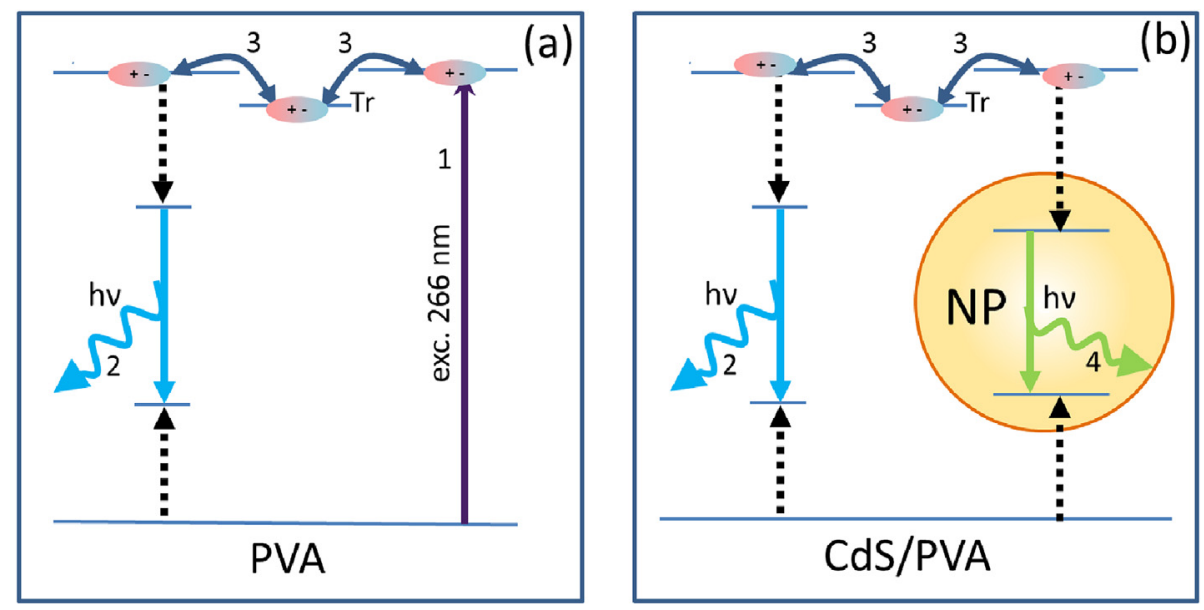

Fig. 4. The scheme of energy transitions in PVA (a) and CdS/PVA nanocomposite (b) that are involved in the afterglow processes.

PVA despite their different emission spectra. The processes that occur in both materials are illustrated by the schemes in Fig. 4, a,b.

The excitation of pure polymer results in the formation of excitons [25] as is shown by the arrow 1 in Fig. 4,a. The excitons recombine and emit light (arrow 2 in Fig. 4,a) while some part of the excitons is captured by traps (the corresponding energy level is labeled $\mathrm{Tr}$ ). The trapped excitons can be thermally released and further participate in the emission processes. The escape of excitons from the traps is a reversible process, thus, an exciton can be several times released and re-trapped (double-sided arrows 3 in Fig. 4, a) before it would recombine radiatively. After switching off the light the trapped excitons are gradually released and cause the afterglow in the same spectral range as the cw luminescence.

Note, that in Fig. 4a,b we show the single level for the traps. However, one should keep in mind that this is done for the simplicity of the scheme. In reality, there is more than one type of traps, as it follows from the discussed above values of the hyperbolic indexes.

At the stationary excitation of composite, the processes are more complicated as compared to the unloaded polymer (Fig. 4, a,b). The excitation can occur via the absorption by polymeric matrix, absorption by NPs, or the excitation can be transferred from the matrix to NPs [15]. The emission of light can occur via two routes that involve the matrix-related (arrow 2) or NP-related energy states (arrow 4) thus causing different shapes of the emission spectra for the polymer and composite (Fig. 1). Similarly to the case of pure matrix, some of the excitons are immobilized on the traps in the matrix, and the dynamic equilibrium is achieved by trapping, releasing and re-trapping processes.

After switching off the light most of the untrapped excitons quickly recombine while the excitons that were accumulated on the traps are gradually released and induce the afterglow of both matrix and embedded NPs, thus, two bands are seen in the transient phosphorescence spectra: the polymer-related emission at $460 \mathrm{~nm}$ and NPs-related emission at $510 \mathrm{~nm}$ (arrows 2 and 4 in Fig. 4,b, respectively). Both emissions decay together with the decay of excitons population on the traps.

\section{Conclusions}

The unusually long low-temperature $(5 \mathrm{~K})$ photoluminescence decays of the CdS/PVA nanocomposite and the pure polymeric matrix were observed. While the spectra of CdS/PVA and PVA are very different, the decays in both materials are characterized by the same average decay time equal to $1.7 \mathrm{~s}$. The time dependencies of the afterglow emission in both cases were fitted by the hyperbolic law using the Becquerel formula with the hyperbolic index of 0.3. The observed long-lasting decay in both structures is suggested to be caused by thermal release of trapped excitons after switching off excitation light and their further participation in the radiative recombination. In both types of the structures, the traps are concluded to be situated within the polymer. In the case of the unloaded polymer, the released excitons recombine within the polymer. In the case of the nanocomposite the released excitons are shared between the matrix and NPs thus producing both the NPrelated and matrix-related afterglow.

\section{Acknowledgements}

Financial support from the Swedish Institute via Visby program and by the Complex program for fundamental research of NAS Ukraine "Fundamental problem of new nanomaterials and nanotechnologies creation" is greatly appreciated.

\section{References}

[1] D. Bera, L. Qian, T.-K. Tseng, P. Holloway, Materials 3 (2010) 2260-2345.

[2] P. Lodahl (Ed.), A Volume in Woodhead Publishing Series in Electronic and Optical Materials, Woodhead Publishing Limited, Cambridge, 2012, pp. 395-420.

[3] H. Zhou, J. Liu, S. Zhang, Trends Anal. Chem. 67 (2015) 56-73.

[4] P. Chin, R. Hikmet, R. Janssen, J. Appl. Phys. 104 (2008) 013108.

[5] J. Cheng, S. Wang, X.-Y. Li, Y. Yan, S. Yang, C.L. Yang, J.N. Wang, W.K. Geial, Chem. Phys. Let. 333 (2001) 375-380.

[6] K. Manzoor, S.R. Vadera, N. Kumara, T.R.N. Kutty, Sol. State Commun. 129 (2004) 469-473.

[7] C. Yoon, H.-G. Hong, H.C. Kim, D. Hwang, D.C. Lee, C.-K. Kim, Y.-J. Kim, K. Lee, Coll. Surf. A Physicochem. Eng. Asp 428 (2013) 86-91.

[8] S. Kango, S. Kali, A. Celli, J. Njugun, Y. Habibi, R. Kumar, A Rev. Prog. Polym. Sci. 38 (8) (2013) 1232-1261.

[9] K. Franz, W. Kehr, A. Siggel, J. Wieczoreck, W. Adam, Luminescent Materials, Ullmann's Encyclopedia of Industrial Chemistry, Wiley-VCH Verlag GmbH \& Co. KGaA, Weinheim, 2000.

[10] M.V. Fok, Introduction to the Kinetics of Luminescence of Crystal Phosphors [in Russian], Nauka, Moscow, 1964.

[11] W. Ma, W. Guo, D. Lu, M. Fan, Y. Wei, Y. Li, S. Shuang, M. Choi, Talanta 116 (2013) 794-800.

[12] Y. Gong, Z. Fan, Sens. Actuators B 202 (2014) 638-644.

[13] Li, Y. Yang, C. Sun, S.-Y. Fu, J. Phys. Chem. C 112 (2008) 17397-17401.

[14] V. Nosenko, G. Rudko, V. Fediv, A. Savchuk, E. Gule, I. Vorona, Nanoscale Res. Lett. 9 (2014) 683-688.

[15] G.Yu Rudko, A.O. Kovalchuk, V.I. Fediv, Q. Ren, W.M. Chen, I.A. Buyanova G. Pozina, Thin Solid Films 543 (2013) 11-15.

[16] G. Rudko, A. Kovalchuk, V. Fediv, W. Chen, I. Buyanova, J. Coll. Interface Sci 452 (2015) 33-37.

[17] S. Santhi, E. Bernstein, Francoise Paille, J. Lumin 117 (2006) 101-112.

[18] G. Ma, S.-H. Tang, W. Sun, Z. Shen, W. Huang, J. Shi, Phys. Lett. A 299 (5-6) 
(2002) 581-585.

19] M. Tanaka, J. Qi, Y Musumoto, J. Cryst. Growth 214 (215) (2000) 410-414.

[20] J. Mieloszyk, R. Drabent, J. Siódmiak, J. Appl. Polym. Sci. 34 (1987) 1577-1580.

[21] U. Woggon, S.V. Bogdanov, O. Wind, K.-H. Schlaad, H. Pier, C. Klingshirn, P. Chatziagorastou, H. Fritz, Phys. Rev. B 48 (16) (1993) 11979-11986.

[22] K. Tomihira, D.G. Kim, M. Nakayama, J. Lumin. 122-123 (2007) 471-473.
[23] E. Becquerel, Firmin Didot, Paris, 1, 1867

[24] V. Degoda, F. Danevich, N. Coron, I. Ivanov, Ya Kogut, E. Makarov, P. de Marcillac, S. Nasonov, L. Torres, V. Tretyak, V. Shlegel, Ya Vasiliev, M. Velazquez, V. Zhdankov, Sol. State Phenom. 230 (2015) 184-192.

[25] K. Ghiggino, C. Barraclough, R.J. Harris, Irradiat. Polym. Mater. Process. Mech. Appl. 527 (1993) 155-166.

Please cite this article in press as: A.O. Kovalchuk, et al., Phosphorescence of CdS nanoparticles in polymer matrix as an indication of host-guest interaction, Materials Chemistry and Physics (2016), http://dx.doi.org/10.1016/j.matchemphys.2016.04.042 